\title{
How to diagnose Mycoplasma pneumoniae etiology in a child with pneumonia?
}

\author{
Matti Korppi
}

Received: 29 June 2011 / Accepted: 7 July 2011 /Published online: 20 July 2011

(C) Springer-Verlag 2011

How to diagnose Mycoplasma pneumoniae etiology in a child with pneumonia?

$\mathrm{Xu}$ et al. compared the detection rates of $M$. pneumoniae in nasopharyngeal aspirates (NPA) vs. bronchoalveolar lavage (BAL) obtained from 406 children hospitalized pneumonia [3]. Serum samples were tested for microbespecific IgM and BAL samples for microbe-specific DNA by quantitative polymerase chain reaction (PCR). NPA samples were studied by the same PCR in $357(88 \%)$ cases. IgM determination was positive in 101 (24.9\%) patients, regarded as mycoplasmal infections and "gold standards" when evaluating the diagnostic value of NPA vs. BAL samples. The positivity rate of PCR was $48.5 \%$ in BAL and $40.9 \%$ in NPA. The sensitivity and specificity of PCR was $78.6 \%$ and $63.5 \%$ in NPA and $70.3 \%$ and $58.7 \%$ in BAL, respectively.

The authors conclude that NPA is superior to BAL for diagnosing mycoplasmal pneumonia [3]. However, there was nearly a total overlapping in the $95 \%$ confidence intervals (CI) of sensitivities and specificities of PCR in NPA vs. BAL. There were some differences in receiver operating characteristic curves, but no comparable parameters like likelihood ratios (LR) or areas under curve were presented. LRs (but not 95\% CIs) can be calculated from the presented figures, and LR+ was 2.15 for PCR in NPA and 1.70 in BAL, which means only minor effects on pretest probability.

The study was retrospective and the material was selected (bronchoscopy needed). The positivity rate in NPA samples was much lower, $16.9 \%$, in all 7,381 pneumonia children treated in the hospital during the study period [3]. In population-based serological studies including both inpatients and outpatients, $M$. pneumoniae has caused $14.3-18.4 \%$ of pneumonia cases in $<5$-year-old and $47.4-54.2 \%$ in $>5$-yearold children [1,2]. Since the positivity rates are dependent on age and setting, the analyses should be age specific, and LR+ and LR- should be used instead of predictive values. In addition, the results are dependent on the "gold standards" used.

\section{References}

1. Don M, Fasoli L, Paldanius $M$ et al (2005) Aetiology of community-acquired pneumonia: Serological results of a paediatric survey. Scand J Infect Dis 37:806-812

2. Korppi M, Heiskanen-Kosma T, Kleemola M (2004) Incidence of community-acquired pneumonia in children caused by Mycoplasma pneumoniae: Serological results of a prospective, population-based study in primary health care. Respirol 9:109-114

3. Xu D, Li S, Chen Z, Du L (2011) Detection of Mycoplasma pneumoniae in different respiratory specimens. Eur J Pediatr 170:851-858
M. Korppi ( $\varangle)$

Pediatric Research Center,

Tampere University and University Hospital,

33014 Tampere, Finland

e-mail: matti.korppi@uta.fi 